\section{Can We Really Skip the Biopsy in Diagnosing Symptomatic Children With Celiac Disease}

$T$ o the Editor: In the March 2013 issue of the Journal of Pediatric Gastroenterology and Nutrition, Klapp et al (1) reviewed 150 symptomatic children with celiac disease, finding that the positive predictive value of applying these new criteria was $97.4 \%$, and concluding that the diagnosis of celiac disease can be accurately made in a selected population without the requirement of a duodenal biopsy, following the new European Society for Pediatric Gastroenetrology, Hepatology, and Nutrition guidelines (2). We performed a multicenter retrospective chart review (3) aimed at determining how many patients would avoid the biopsy and at verifying potential drawbacks of eliminating endoscopy.

A total of 279 symptomatic patients (mean age 8.6 years) from 4 US centers had data available for both tissue transglutaminase-immunoglobulin A (tTG) and endomysium antibodies-immunoglobulin A. One hundred twenty-six patients $(45 \%)$ had tTG $>10 \times$ normal and positive EMA. Of these 126 patients, 115 (91\%) underwent endoscopy with biopsies. Pathology reports showed all of them to have celiac disease ( $74 \%$ Marsh 3); however, $10 \%$ of them had unexpected additional diagnoses: chronic gastritis (2), reflux esophagitis (5), esophagitis (2), eosinophilic esophagitis (1), and Helicobacter pylori (1). Sixteen additional asymptomatic patients in our series also had tTG $>10 \times$ normal and positive endomysium antibodies-immunoglobulin A. Of them, only 1 had an unexpected diagnosis $(\mathrm{H}$ pylori).

Thus, although we also found that a large portion of our patients would have been properly diagnosed as having celiac disease without a biopsy, by avoiding the diagnostic procedure, additional, unexpected diagnoses would have been missed in $>10 \%$ of such symptomatic patients. We urge careful follow-up of symptomatic patients after they are diagnosed without a biopsy to avoid overlooking associated conditions.

\section{${ }^{*}$ Stefano Guandalini and ${ }^{\dagger}$ Catherine Newland ${ }^{*}$ University of Chicago Celiac Disease Center ${ }^{\dagger}$ University of Chicago, Chicago, $I L$}

\section{REFERENCES}

1. Klapp G, Masip E, Bolonio M, et al. Coeliac disease: the new proposed ESPGHAN diagnostic criteria do work well in a selected population. J Pediatr Gastroenterol Nutr 2013;56:251-6.

2. Husby S, Koletzko S, Korponay-Szabo IR, et al. ESPGHAN guidelines for the diagnosis of celiac disease in children and adolescents: an evidence-based approach. J Pediatr Gastroenterol Nutr 2012;54:136-60.

3. Newland C, Reilly N, Bracken J, et al. A multicenter review of the new ESPGHAN guidelines for coeliac disease: how many biopsies can we really skip? Manuscript submitted.

\section{Authors' Response}

\footnotetext{
$T$ $o$ the Editor: Guandalini and Newland express concern that other diagnoses of the upper gastrointestinal tract will be missed if the diagnosis of celiac disease (CD) is made without performing an upper endoscopy. We thank the authors for pointing out this issue and the journal for affording us the chance to respond.

The authors report the results of their retrospective chart review with endoscopic findings in 115 children with anti-TG2
}

antibody concentrations $>10$ times the upper limit of normal. According to the new European Society for Pediatric Gastroenterology, Hepatology, and Nutrition (ESPGHAN) guidelines on the diagnosis of $\mathrm{CD}$, these patients would qualify for the option to omit the biopsies under the following conditions in addition to the high TG2 antibody concentrations: symptoms suggestive of CD, positive anti-endomysial antibodies in a second blood sample, HLA-DQ2 or -DQ8 positivity, and informed consent after discussion with a pediatric gastroenterologist (1). In 11 of 115 patients, abnormal findings were reported; however, we would consider some of them less relevant or part of the celiac pathology. For example, Helicobacter pylori infection does not need to be treated in the absence of erosions and ulcerations (2). Chronic superficial or lymphocytic gastritis are common findings in children with untreated CD $(3,4)$ and should resolve with a gluten-free diet. The remaining patients had different types of esophagitis, one of them with eosinophilic infiltrations. No information is provided as to whether the diagnosis of esophagitis was based on macroscopic findings with mucosal breaks (erosions, ulcerations) or histology only.

Untreated CD causes dysmotility with delayed gastric emptying because of reduced secretion of gastrointestinal hormones in the presence of enteropathy. This may predispose to gastroesophageal reflux and esophagitis, but again, dysmotility will resolve after normalization of the small bowel mucosa with a gluten-free diet. In addition, it is unclear how many unselected control children would show any esophageal pathology. The Kalixanda study reports findings in 1000 population-based adults who underwent upper endoscopy with multiple biopsies. Even in the absence of any symptoms, $9.5 \%$ were found to have erosive esophagitis. This proportion reached $20 \%$ to $36 \%$ in individuals complaining of occasional to daily symptoms (5). In the same study, definite or probable eosinophilic esophagits (EoE) was identified in $1.1 \%$, and eosinophilic infiltrations were related to reflux esophagitis (6). It has been suggested that EoE is more frequent than expected in patients with $\mathrm{CD}$; however, a recent meta-analysis on incidence and prevalence of EoE in children reported a prevalence ranging from $1.1 \%$ to $5.1 \%$ in CD compared with $2.1 \%$ to $4.9 \%$ in children who underwent endoscopy for abdominal pain (7).

The data point to the dilemma that a concurrent disease may be responsible for the symptoms leading to medical attention, and CD essentially may be asymptomatic. Symptomatology and clinical judgment are important elements of the ESPGHAN guidelines. In cases in which the type of gastrointestinal symptoms (mainly abdominal pain) indicates the possibility of additional pathology, it is at the discretion of the managing physician to perform an endoscopy already at the beginning of the clinical workup. Although CD cannot be diagnosed after starting the diet, an endoscopical search for additional pathology can be performed at any later time in those children whose symptoms do not respond to a gluten-free diet. This would still spare a majority from the invasive procedure.

In conclusion, we believe that the findings observed by Guandalini and Newland do not justify changing the ESPGHAN guidelines and the option to omit duodenal biopsies. At the same time, we strongly support their recommendation to follow-up on these children receiving a gluten-free diet and evaluate those in whom symptoms do not resolve.

${ }^{*}$ Steffen Husby, ${ }^{\dagger}$ Sibylle Koletzko, and ${ }^{\ddagger}$ Ilma Korbonay-Szabo *Hans Christian Andersen Children's Hospital, Odense University Hospital, Denmark ${ }^{\dagger}$ University of Munich Medical Center, Dr von Hauner Children's Hospital, Munich, Germany

${ }^{\ddagger}$ University of Debrecen, Medical and Health Science Center, Debrecen, Hungary 


\section{REFERENCES}

1. Husby S, Koletzko S, Korponay-Szabo IR, et al. European Society for Pediatric Gastroenterology, Hepatology, and Nutrition guidelines for the diagnosis of coeliac disease. J Pediatr Gastroenterol Nutr 2012;54:13660 .

2. Koletzko S, Jones NL, Goodman KJ, et al. Evidence-based guidelines from ESPGHAN and NASPGHAN for Helicobacter pylori infection in children. J Pediatr Gastroenterol Nutr 2011;53:230-43.

3. Bhatti TR, Jatla M, Verma R, et al. Lymphocytic gastritis in pediatric celiac disease. Pediatr Dev Pathol 2011;14:280-3.

4. Nenna R, Magliocca FM, Tiberti C, et al. Endoscopic and histological gastric lesions in children with celiac disease: mucosal involvement is not only confined to the duodenum. J Pediatr Gastroenterol Nutr 2012;55:728-32.

5. Ronkainen J, Aro P, Storskrubb T, et al. Gastro-oesophageal reflux symptoms and health-related quality of life in the adult general population-the Kalixanda study. Aliment Pharmacol Ther 2006; 23:1725-33.

6. Ronkainen J, Talley NJ, Aro P, et al. Prevalence of oesophageal eosinophils and eosinophilic oesophagitis in adults: the population-based Kalixanda study. Gut 2007;56:615-20.

7. Soon IS, Butzner JD, Kaplan GG, et al. Incidence and prevalence of eosinophilic esophagitis in children: systematic review and meta-analysis. J Pediatr Gastroenterol Nutr 2013;57:72-80.

\section{Antitumor Necrosis Factor, Infliximab, and Adalimumab: Use With Caution in Eosinophilic Bowel Disease}

\footnotetext{
$T$
} o the Editor: We read the article by Turner et al (1) with great interest and concern because it proposes that "infliximab and adalimumab may be effective in refractory idiopathic eosinophilic enterocolitis" (EEC). Our concerns lie in the lack of diagnostic clarity, uncertainty of clinical efficacy of the treatments, the suggested chronic use of medications with significant potential adverse effects, and uncertain therapeutic rationale. Histological features defining eosinophilic gastrointestinal disease beyond the esophagus remain difficult to define, and parameters defining EEC are even less clear. The authors quote 3 review references that provide no diagnostic clarity regarding EEC (2-4). Because images of histological slides were not included, the histological parameters used to define EEC in the presented patients remain unclear.

We are uncertain whether the pain experienced can be clearly ascribed to the duodenal eosinophilia or vice versa because it relates to the 5 responsive patients. Three patients showed a decrease in duodenal eosinophil numbers, 1 remained the same, and 1 was not reassessed. Although 3 patients experienced reduction in pain and a decrease in eosinophilia, the cause and effect are difficult to ascertain because there is a significant placebo effect associated with abdominal pain. Systemic and topical steroids, medications known to decrease mucosal eosinophilia, did not reduce symptoms in 3 of 5 of these patients. A further confounder is the long time intervals that occurred in some of the patients between initiating the biological treatments and obtaining the mucosal findings; this raises the question of how the mucosa appeared at the beginning of biological treatment.

The long treatment duration and lack of documented toxicity monitoring are concerning because they appear to support the use of these medications at "higher doses than practiced for IBD" for up to 6 years without significant risk (5). Although there is a small body of literature suggesting that TNF- $\alpha$ is a therapeutic target for eosinophilic esophagitis, evidence supporting its role in other eosinophilic gastrointestinal diseases is lacking (6).

The authors suggest that additional studies are warranted; however, we propose that until more supportive evidence becomes available, these medications be used with extreme caution for welldefined time periods and accompanied by close monitoring for adverse effects.

${ }^{*}$ Glenn T. Furuta, ${ }^{\dagger}$ Dan Atkins, ${ }^{\ddagger}$ Kelley E. Capocelli
${ }^{*}$ Edwin F. de Zoeten, ${ }^{\dagger}$ David Fleischer, and
${ }^{*}$ Calies Menard-Katcher
${ }^{*}$ Section of Pediatric Gastroenterology, Hepatology, Digestive
Health Institute, Children's Hospital Colorado
${ }^{\dagger}$ Department of Pediatrics
${ }^{\ddagger}$ Department of Pathology, National Jewish Health, Aurora, CO

\section{REFERENCES}

1. Turner D, Wolters VM, Russell RK, et al. Anti-TNF, infliximab, and adalimumab can be effective in eosinophilic bowel disease. $J$ Pediatr Gastroenterol Nutr 2013;56:492-7.

2. Yan BM, Shaffer EA. Primary eosinophilic disorders of the gastrointestinal tract. Gut 2009;58:721-32.

3. Khan S, Orenstein SR. Eosinophilic gastroenteritis: epidemiology, diagnosis and management. Paediatr Drugs 2002;4:563-70.

4. Alfadda AA, Storr MA, Shaffer EA. Eosinophilic colitis: an update on pathophysiology and treatment. Br Med Bull 2011;100:59-72.

5. Ghabril M, Bonkovsky HL, Kum C, et al. Liver injury from tumor necrosis factor-alpha antagonists: analysis of thirty-four cases. Clin Gastroenterol Hepatol 2013;11:558-564e3.

6. Straumann A, Bussmann C, Conus S, et al. Anti-TNF-alpha (infliximab) therapy for severe adult eosinophilic esophagitis. J Allergy Clin Immunol 2008;122:425-7.

\section{Authors' Response}

$T$ $o$ the Editor: We thank Furuta et al for the opportunity to clarify our standpoint regarding the possible effectiveness of antitumor necrosis factor (anti-TNF) in eosinophilic enterocolitis (EEC).

Certainly, case reports should not be used to change clinical practice. They are, however, a rich source of hypotheses to be tested in further studies of more robust design. Our reported children had poor quality of life because of severe ongoing symptoms despite treatment attempts with all of the standard interventions. In these extreme cases, one often considers unconventional treatments, despite the lack of level I evidence. Specifically in EEC, which is extremely rare, its pathophysiology is totally obscure, and all suggested treatments to date have been based on case series only.

Despite the inherent biases and limitations of our case series, which we tried to minimize, there is more to suggest that treatment with anti-TNF was beneficial. Response to the drug was rapid and obvious in the responsive cases, in a similar manner to what we see in IBD. When available, repeated biopsies did show marked improvement, and the loss of response to infliximab was in decreasing intervals from the previous infusions, lending support to a true biological effect of the drug. All of the cases were previously 
refractory to multiple aggressive therapies and there is no reason to believe that suddenly a placebo effect will be so striking only with anti-TNF.

We do not suggest that anti-TNF should be routinely used in EEC. We fully agree with Furuta et al that a trial of anti-TNF treatment in EEC should only be considered at this time in severe and resistant cases, accompanied by monitoring for adverse effects as we practice in classic IBD. Response should be assessed closely (including repeated biopsies) to ensure that the drug is indeed effective. It is also reasonable to plan exit strategies in the responsive cases. Indeed, the literature is eagerly awaiting level I evidence for effective therapies and "diagnostic clarity" of EEN, but until then, we must still treat our patients, making use of the bestavailable evidence.

${ }^{*}$ Dan Turner and ${ }^{\dagger}$ Craig Friesen

* Jerusalem, Israel

${ }^{\dagger}$ Kansas City, $M O$ 